\title{
Verification of the effect of yarn torsional rigidity on fabric bending
}

\section{rigidity in any direction}

\author{
Julie Peiffer ${ }^{1}$, KyoungOk Kim², Masayuki Takatera ${ }^{2}$
}

${ }^{1}$ Department of Bioscience and Textile Technology, Interdisciplinary Division of Science and Technology, Shinshu University, 3-15-1 Tokida, Ueda, Nagano 386-8567, Japan.

${ }^{2}$ Division of Kansei and Fashion Engineering, Institute for Fiber Engineering (IFES), Interdisciplinary Cluster for Cutting Edge Research (ICCER), Shinshu University, 3-15-1 Tokida, Ueda, Nagano 386-8567, Japan

takatera@shinshu-u.ac.jp

\begin{abstract}
The effect of yarn torsional rigidity was verified on the Cooper model for fabric bending rigidity in any direction. We made five cotton fabrics with different weft densities and prepared three commercial fabrics as experimental samples. The torsional rigidity of yarn from the bobbin and that of yarn directly extracted from fabric were measured with a yarn torsional tester. The bending rigidity of yarn from the bobbin was measured using the same pure bending tester as used in fabric bending testing. The bending rigidity of thin fabric was calculated using torsional rigidities of yarns extracted from the fabric and showed better agreement with the experimental values than that calculated using the torsional rigidity of yarn from the bobbin. Indeed, measurements showed that the torsional rigidity of yarn from the bobbin was appreciably higher than the torsional rigidity of yarn from the fabric. This is due to the crimp in the yarn. The fabric bending rigidity can be predicted using the Cooper model with torsional rigidities of yarns extracted from the fabric.
\end{abstract}

KEYWORDS: yarn torsional rigidity, bending rigidity, fabric, yarn, anisotropy

\section{Introduction}

When designing clothing and furniture, the bending properties of fabric are important because they affect the hand and draping behavior.. The bending of fabric must therefore be considered when designing products. The hand and draping properties are directly linked to the bending properties of the fabric and yarn. If fabric bending properties can be predicted only using the yarn properties, appropriate yarn can be selected according to the fabric intended use. Additionally, fabric is an anisotropic material, and thus its properties in all directions should be considered.

Numerous studies have measured the bending rigidity and bending hysteresis of fabric. The quantitative evaluation of the bending behavior of woven fabric started with the work of Pierce [1], who developed a method of measuring bending rigidity under low stress. Grosberg [2] showed that the fabric bending behavior depends on two factors: the bending rigidity and the frictional restraint couple. Pierce was the first to construct a model that could be used to estimate a fabric's bending stiffness in any direction from the anisotropic Young's modulus for fabric. Go et al. [3] elaborated a bending stiffness model for woven fabric using the bending rigidity of yarn without torsional rigidity, and neglecting the crimp effect. Cooper [4] established a 
bending model for any direction taking into account the effect of the torsional rigidity on the bending behavior of fabric. Chapman and Hearle [5] also investigated a method of predicting bending rigidity using an energy method and ultimately obtained the same model as Cooper. Shinohara et al. [6] went further to explain the torsional component of Cooper's model. They showed the effect of yarn torsional rigidity on fabric bending in the bias direction qualitatively. However, the effect of yarn torsional rigidity on fabric bending rigidity has not been clarified experimentally. Yarn bending and torsional rigidities have to be measured to validate the model.

The measurement of yarn properties is difficult owing to the small torque and bending moment in addition to unevenness. The first yarn bending measurement was carried out by Pierce [1] employing a cantilever method also used for fabrics. Saxl [7], Horio and Onogi [8] and other researchers have developed methods of measuring the bending rigidity of yarns. A pure bending tester for fabric and yarn was developed by Isshi [9], opening the door for the development of a modern apparatus. The Kawabata Evaluation System (KES) FB-2 for pure bending is currently used to test fabric and yarns [10].

The yarn torque can be measured using a torsion pendulum but the results can be distorted by a damping factor [11]. Measurements using a torsional tester, such as a galvanometer, are also common [12]. Furthermore, KES-YN1 is used to obtain the yarn torsional rigidity [13-15].

The simulation of fabric properties using yarn properties is carried out around the world [16-20]. When simulating fabric bending behavior, it is important to take into account yarn properties such as tensile, transverse compression, bending and torsion properties. Current simulations use the estimated or measured yarn tensile modulus, while bending and torsional moduli are calculated from a tensile modulus assumed to be isotropic. However, yarns are discontinuous and anisotropic, and bending and torsional moduli are thus independent of the tensile modulus. It is necessary to include those effects in a future model in realizing a more accurate simulation.

In this study, the effect of yarn torsional rigidity on fabric bending rigidity in any direction was verified experimentally. The fabric bending rigidity was calculated using the measured yarn bending and torsional rigidities and compared the results with the bending rigidity of fabric measured in several directions. Furthermore, the effect of crimp on the torsional rigidity of yarn was discussed.

\section{Theoretical}

Cooper [4] proposed a mathematical model with which to calculate the bending rigidity of woven fabric in any direction according to the yarn bending rigidity and torsional component. Shinohara et al. [6] then clarified the model to get the torsional component from the yarn torsional rigidity and yarn density.

In Cooper's model, the bending rigidity in warp and weft is estimated using the relation

$$
B_{f}=n B_{y} \text {, }
$$

where $B_{f}$ is the bending rigidity of the fabric in $\mathrm{N} \cdot \mathrm{cm}^{2} / \mathrm{cm}, n$ is the yarn density in the testing direction in yarns $/ \mathrm{cm}$, and $B_{y}$ is the bending rigidity of a yarn in $\mathrm{N} \cdot \mathrm{cm}^{2}$. The bending rigidity of the fabric can then be estimated for any direction using

$$
\left(B_{f}\right)_{\alpha}=B_{f 1} \cdot \cos ^{4} \alpha+B_{f 2} \cdot \sin ^{4} \alpha+\left(J_{1}+J_{2}\right) \cos ^{2} \alpha \sin ^{2} \alpha,
$$

where $\alpha$ is the angle of the fabric from the warp, $J$ is the effective torsional rigidity per yarn of the warp and weft assemblies as they exist in the fabric structure, and the subscripts 1 and 2 respectively refer to the warp and weft directions. 
Shinohara et al. [6] then explained the $J$ term by showing that it could be defined as $J=J_{y} \cdot n$, where $J_{y}$ is the yarn torsional rigidity in $\mathrm{N} \cdot \mathrm{cm}^{2} / 2 \pi$. Therefore,

$$
\left(B_{f}\right)_{\alpha}=B_{f 1} \cdot \cos ^{4} \alpha+B_{f 2} \cdot \sin ^{4} \alpha+\left(n_{1} \cdot J_{y 1}+n_{2} \cdot J_{y 2}\right) \cos ^{2} \alpha \sin ^{2} \alpha .
$$

However, Cooper and Shinohara et al. did not provide an experimental verification with the torsional rigidity of yarn.

When the same yarn is used for both warp and weft, Equation (3) can be simplified as

$$
\left(B_{f}\right)_{\alpha}=B_{y} \cdot\left(n_{1} \cdot \cos ^{4} \alpha+n_{2} \cdot \sin ^{4} \alpha\right)+J_{y} \cdot\left(n_{1}+n_{2}\right) \cos ^{2} \alpha \sin ^{2} \alpha,
$$

where $B_{y}$ is the bending rigidity of the yarn and $J_{y}$ is the torsional rigidity of the yarn.

\section{Experimental}

To verify the relationship between yarn rigidities and bending properties of a fabric in various directions, yarn bending and torsional rigidities were measured and the fabric bending rigidity was calculated using Equation (3) and the results were compared with the bending rigidity of fabric measured in several directions. To investigate the effect of crimp, the torsional rigidity of straight yarns from a bobbin was compared with that of crimped yarn extracted from fabric.

\section{Measurement of yarn torsional rigidity and fabric and yarn bending rigidities}

Yarn torsional rigidity

To obtain torsional rigidity, a KES-YN1 yarn torsional tester (Kato Tech Co. Ltd. Kyoto, Japan) was used as shown in Figure 1. This device allows the measurement of the twist angle and torque of the yarn. In the tester, the yarn is placed between two clamps, one movable and one fixed. The movable part of the apparatus then makes a $6 \pi$ rotation in one direction and then a $12 \pi$ rotation in the other, and returns to its original position while the device registers the torque and twist angle. Torsional rigidity is obtained from the mean of the two slopes between $2 \pi$ and $4 \pi$ in forward rotation and between $-2 \pi$ and $-4 \pi$ in backward rotation. The rotating speed was $\pi / 15 \mathrm{~s}^{-1}$. An example of a torque-twist angle curve is shown in Figure 2. The asymmetry is due to the nature of the yarn. The torque will be higher when rotating in the direction of the twist, because it increases the twist, while rotating in the opposite direction of the twist will untwist the yarn.

To minimize yarn tension in the torsional test, the yarn sample was subjected only to the weight of the clamp $(0.342 \mathrm{~g})$ and its own weight during the testing process. The effective sample length was $3 \mathrm{~cm}$. To test crimped yarns, an additional weight of $\left(0.2 \cdot T_{\text {tex }}+4\right) \mathrm{cN}$ was added, where $T_{\text {tex }}$ is the yarn count in tex, when making the $3 \mathrm{~cm}$ length yarn samples to obtain a constant crimp according to the Japanese Industrial Standards (JIS) for testing fabrics (JIS L1096:2010 [21]). Figure 3 shows a sample layout. For bobbin yarn, to guarantee a constant length for all samples, a preload equivalent to the weight of 200 meters of yarn was applied when making a sample. Then, after 24 hours under standard conditions (temperature of $20 \pm 1{ }^{\circ} \mathrm{C}$ and relative humidity of $65 \% \pm 5 \%$ ), the yarn torsional rigidity was measured. Twenty-five samples for each kind of yarn were prepared and then tested. 


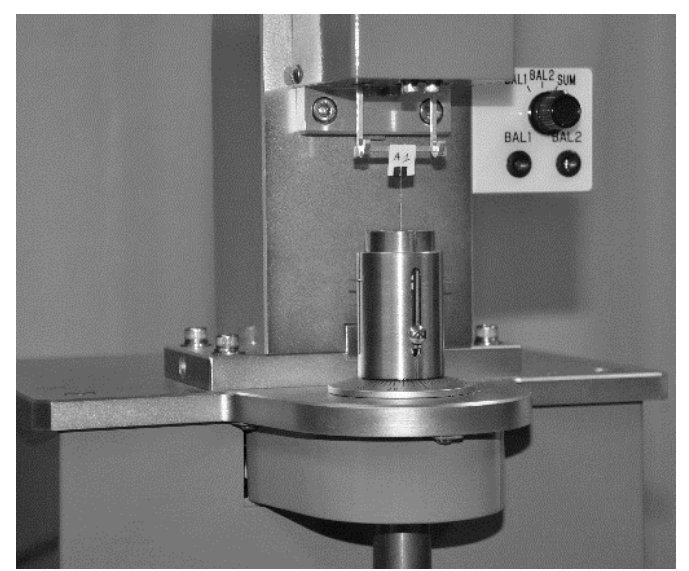

Figure 1. Yarn torsional testing with a KES-YN1 device

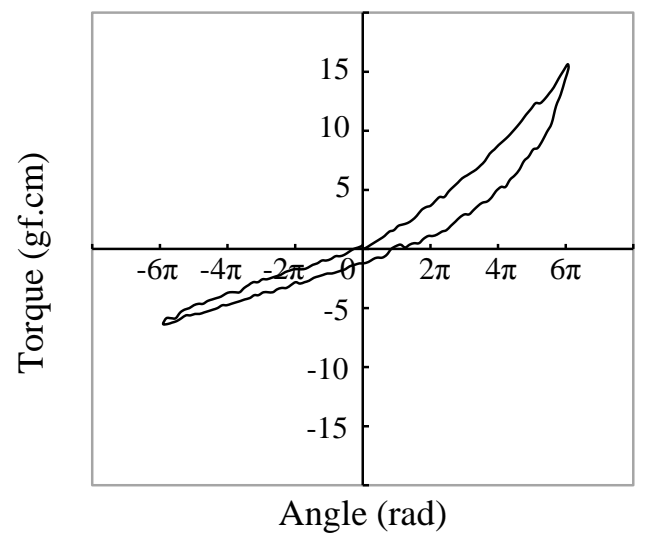

Figure 2. Typical torque curve for spun yarn

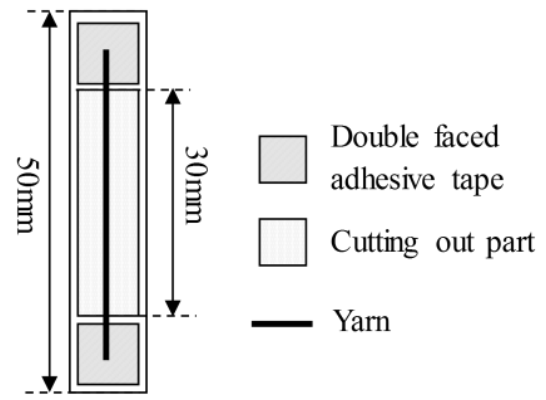

Figure 3. Sample layout

\section{Yarn bending rigidity}

Yarn bending rigidity was measured using the Kawabata KES-FB2 pure bending tester (Kato Tech Co. Ltd., [10]). The bending rigidity is obtained from the mean of the two slopes between curvatures of 0.5 and 1.5 $\mathrm{cm}^{-1}$ in the forward direction and between -0.5 and $-1.5 \mathrm{~cm}^{-1}$ in the backward direction. Cotton yarn has low bending rigidity and does not have a homogeneous structure along its length, and it is thus difficult to accurately obtain the bending rigidity for a single yarn. Therefore, usually, multiple yarns distributed with densities of 10-20 yarns/cm over $2 \mathrm{~cm}$ were tested together as shown in Figure 4 . For the yarn torsional rigidity samples, a weight equivalent to $200 \mathrm{~m}$ of the yarn was applied during the placing of the yarn to guarantee a constant length for all yarns. To obtain more accurate measurements, 100 and 200 yarn samples distributed on $10 \mathrm{~cm}$, as shown in Figure 5, were tested. The two had similar accuracy, andthus it was decided to prepare samples of 100 yarns. An example of a yarn bending rigidity curve is shown in Figure 6. Fifteen samples per kind of yarn were prepared. After being conditioned for 24 hours under the standard conditions, the samples were tested.

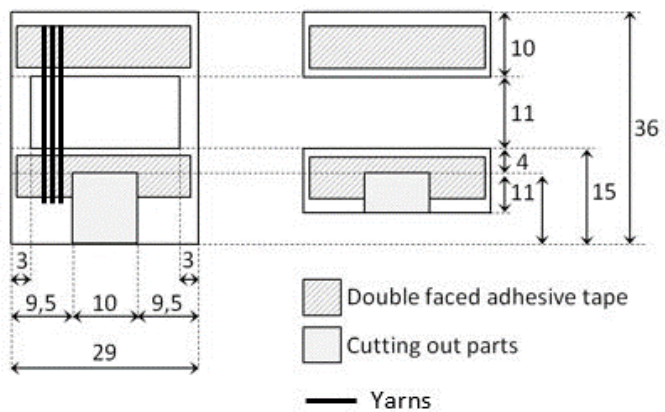

(a) Yarn bending sample layout

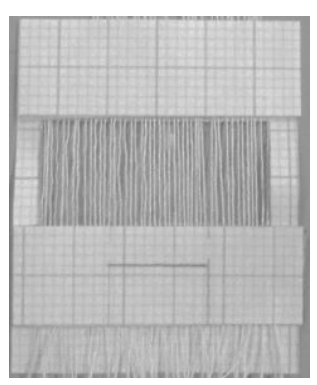

(b) Yarn bending sample (before test) 
Figure 4. Yarn bending samples for a $2 \mathrm{~cm}$ width

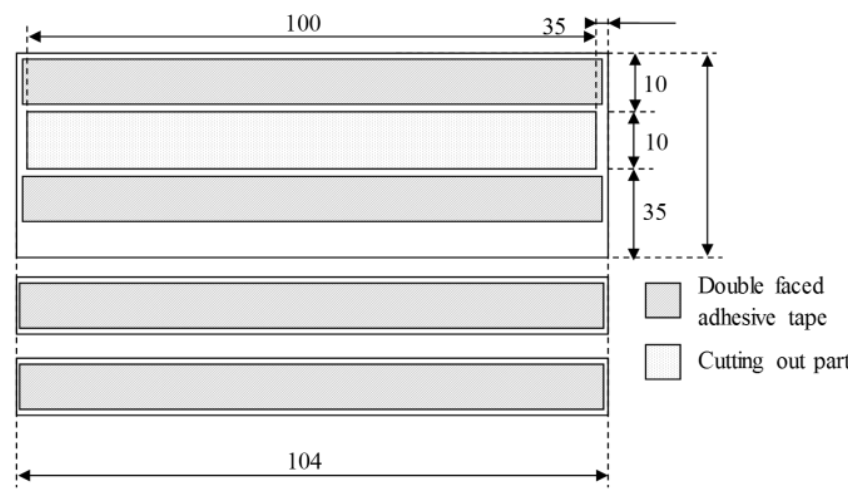

(a) Yarn bending sample layout

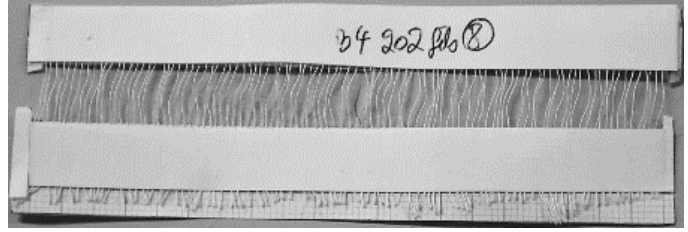

(b) Yarn bending sample (after test)

Figure 5. Yarn bending samples for a $10 \mathrm{~cm}$ width

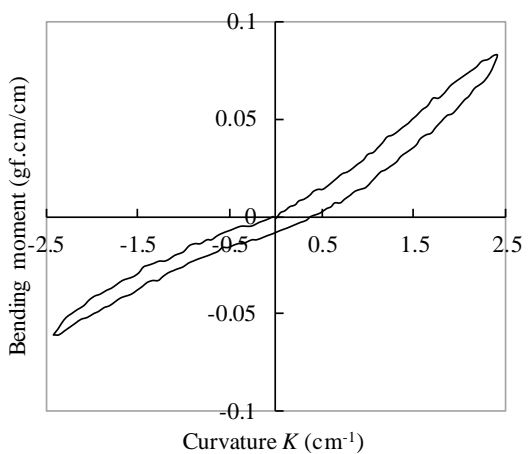

Figure 6. Typical bending moment curve of yarn $(20$ yarns $/ \mathrm{cm})$

\section{Fabric bending rigidity}

A Kawabata KES-FB2 pure bending tester (Kato Tech Co. Ltd., [10]) was used to measure the bending rigidity of the fabric samples. For the fabric bending measurement, $20 \mathrm{~cm} \times 20 \mathrm{~cm}$ samples were prepared along the warp, along the weft, and at $22.5^{\circ}, 45^{\circ}$, and $67.5^{\circ}$ from the warp $\left(0^{\circ}\right)$. Five samples were prepared for each testing direction. Then, after conditioning the samples for 24 hours under the standards conditions, the bending rigidity of the samples was measured.

\section{Test materials}

Eight kinds of plain woven cotton fabric samples were prepared/tested, as shown in Table 1. The fabrics for samples A to E were made using the same cotton non-sized yarn in the warp and weft, and using the same weaving loom. Only the weft density was changed for each sample. For samples A to E, both raw yarn from a bobbin and extracted yarns were used in testing the torsional and bending rigidities to compare the crimp effect. Cotton yarn for samples A to E were made in the same conditions, and the twist factor was $K=5.0$. The high volume instrument (HVI) data for the cotton used gave an average length of $36.6 \mathrm{~mm}$ and a fineness of 0.15 tex.

The fabrics for samples $\mathrm{F}$ to $\mathrm{H}$ were commercial products. For samples $\mathrm{F}$ to $\mathrm{H}$, yarns were directly extracted from the fabric for yarn testing. In yarn bending tests, $10 \mathrm{~cm}$-wide samples were used for samples A to E, and $2 \mathrm{~cm}$-wide samples were used for samples $\mathrm{F}$ to $\mathrm{H}$.

Table 1. Fabric and yarn specifications

\begin{tabular}{|c|c|c|c|c|c|c|c|c|}
\hline $\begin{array}{ll}\text { Characteristics } & \text { Sample } \\
\end{array}$ & A & B & $\mathrm{C}$ & $\mathrm{D}$ & $\mathrm{E}$ & $\mathrm{F}$ & G & $\mathrm{H}$ \\
\hline Weave & \multicolumn{8}{|c|}{ Plain weave } \\
\hline Material & \multicolumn{8}{|c|}{ Cotton } \\
\hline
\end{tabular}




\begin{tabular}{|c|c|c|c|c|c|c|c|c|}
\hline Area density $\left(\mathrm{g} / \mathrm{m}^{2}\right)$ & 111.8 & 129.1 & 130.9 & 140.2 & 150.4 & 148.8 & 81.0 & 47.9 \\
\hline Warp weave density (ends/cm) & \multicolumn{5}{|c|}{23.6} & 28 & 28 & 20 \\
\hline Weft weave density (picks/cm) & 19.7 & 23.6 & 27.6 & 31.5 & 35.4 & 23 & 26 & 14 \\
\hline Warp crimp (\%) & 21.8 & 15.6 & 16.9 & 12.4 & 11.7 & 4.8 & 3.5 & 1.7 \\
\hline Weft crimp (\%) & 12.7 & 14.7 & 16.5 & 16.7 & 18.5 & 21.0 & 7.0 & 7.0 \\
\hline Warp yarn count (tex) & \multicolumn{5}{|c|}{20} & 30 & 16 & 14 \\
\hline Weft yarn count (tex) & \multicolumn{5}{|c|}{20} & 30 & 14 & 14 \\
\hline Origin & \multicolumn{5}{|c|}{ Produced product } & \multicolumn{3}{|c|}{ Commercial product } \\
\hline Yarn twist(tpm)(twist factor) & \multicolumn{5}{|c|}{$1069(K=5.0)$} & \multicolumn{3}{|c|}{-} \\
\hline Fiber average length(mm) & \multicolumn{5}{|c|}{36.6} & \multicolumn{3}{|c|}{ - } \\
\hline Fiber average fineness (tex) & \multicolumn{5}{|c|}{0.15 . } & \multicolumn{3}{|c|}{ - } \\
\hline
\end{tabular}

\section{Results and Discussion}

Table 2 gives the measured bending rigidities of yarns. Figure 7 compares the torsional rigidities of yarns extracted from the fabric with the torsional rigidity of bobbin yarns. The torsional rigidities of yarns extracted from the fabric are very similar among all produced samples, even if the weft density differed. However, there was a large difference from the torsional rigidity of yarn taken directly from the bobbin. This could be due to crimp. Table 3 gives the measured torsional rigidities of yarns for samples A to C.

Table 2. Measured bending rigidities of yarns $\left(\mu \mathrm{N} \cdot \mathrm{cm}^{2}\right)$

\begin{tabular}{|c|c|c|c|}
\hline \multicolumn{2}{|c|}{ Bending rigidity of one yarn } & Mean & SD \\
\hline \multicolumn{2}{|c|}{ A-E (from bobbin) } & 16.09 & 0.03 \\
\hline \multirow{2}{*}{ F (from fabric) } & warp & 0.277 & 0.011 \\
\cline { 2 - 4 } & weft & 0.248 & 0.011 \\
\hline \multirow{2}{*}{ G (from fabric) } & warp & 0.321 & 0.008 \\
\cline { 2 - 4 } & weft & 0.179 & 0.008 \\
\hline \multirow{2}{*}{ H (from fabric) } & warp & 0.694 & 0.019 \\
\cline { 2 - 4 } & weft & 0.154 & 0.006 \\
\hline
\end{tabular}

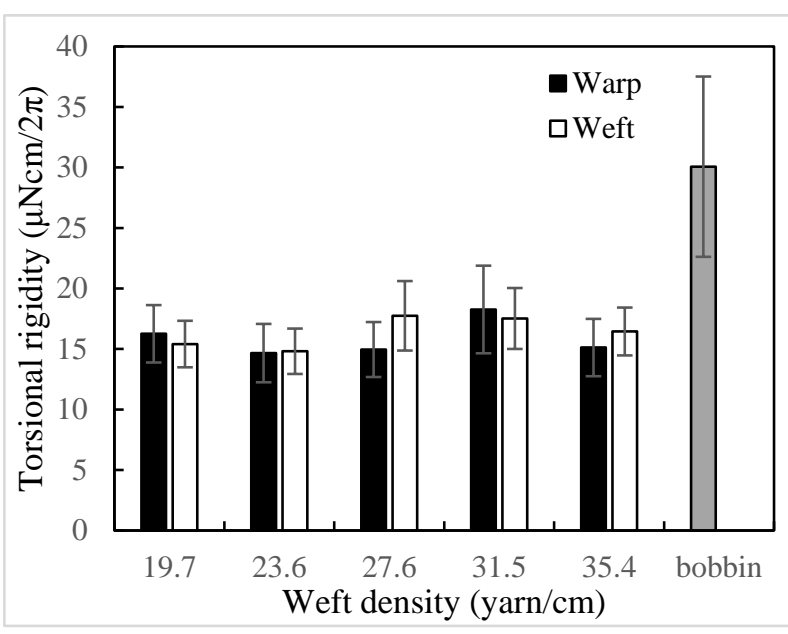

Figure 7. Measured torsional rigidities of yarns extracted from fabric for samples A to E

Table 3. Measured torsional rigidities of yarns for samples $\mathrm{F}$ to $\mathrm{H}$

\begin{tabular}{|c|c|c|}
\hline \multirow{2}{*}{ Sample } & \multicolumn{2}{|c|}{ Torsional rigidity $\left(\mu \mathrm{N} \cdot \mathrm{cm}^{2} / 2 \pi\right)$} \\
\cline { 2 - 3 } & Warp & Weft \\
\hline
\end{tabular}




\begin{tabular}{|c|c|c|c|c|}
\hline & Mean & SD & Mean & SD \\
\hline F & 34.20 & 3.78 & 28.93 & 4.22 \\
\hline G & 32.02 & 8.42 & 12.99 & 0.91 \\
\hline H & 65.95 & 2.50 & 21.30 & 3.40 \\
\hline
\end{tabular}

With the measured values of yarn properties, Equation (3) was verified. Two measurements of each yarn's torsional rigidity were used in the calculating the fabric bending rigidities: one for a yarn directly taken from the bobbin and the other for yarn extracted from each fabric sample in both the warp and weft. The experimental and calculated fabric bending rigidities are compared in Figures 8 to 15.

Because the yarn torsional rigidity did not affect the bending rigidity in the yarn direction, errors in warp and weft direction are due to the measured yarn bending rigidity. Samples C, D and E have good agreement in yarn directions, but samples A and B are slightly different. Figure 16 shows bending rigidity per yarn calculated from fabric bending rigidity using Equation (1), and measured yarn bending rigidity from the bobbin for samples A to E. It was found that the calculated yarn bending rigidities of lower weft densities are lower than those of higher density fabrics, especially in the weft direction. Samples A and B had the lowest weft density, and their calculated bending rigidities of yarn were lower than the measured yarn bending rigidity. This caused the difference in the calculated fabric bending rigidities for warp and weft directions.

In bias directions of samples A to $\mathrm{E}$, the calculated bending rigidity with yarns extracted from the fabric had better agreement than that with the yarn taken from the bobbin. In the calculation of bending rigidity with yarn from the bobbin, the error was a maximum in the $45^{\circ}$ direction. This error is clearly explained by the $J_{y}$ value in Equation (4). Figure 7 shows that yarn torsional rigidities extracted from fabric were significantly lower than yarn torsional rigidity of yarn from the bobbin. This could be due to the crimp effect.

If the torsional rigidity of yarn directly extracted from the bobbin is measured, the fabric bending rigidity can be calculated before weaving. However, the results show that better results are obtained using the torsional rigidity of extracted yarns.

Samples F, G and $\mathrm{H}$ are commercial products and yarn on the bobbin was not available. Thus only the properties measured for the yarn extracted from the fabric were used. The three samples had good agreement between calculated and experimental fabric bending rigidity, especially in the cases of samples $\mathrm{G}$ and $\mathrm{H}$. This result confirms the previous results obtained for samples A to E.
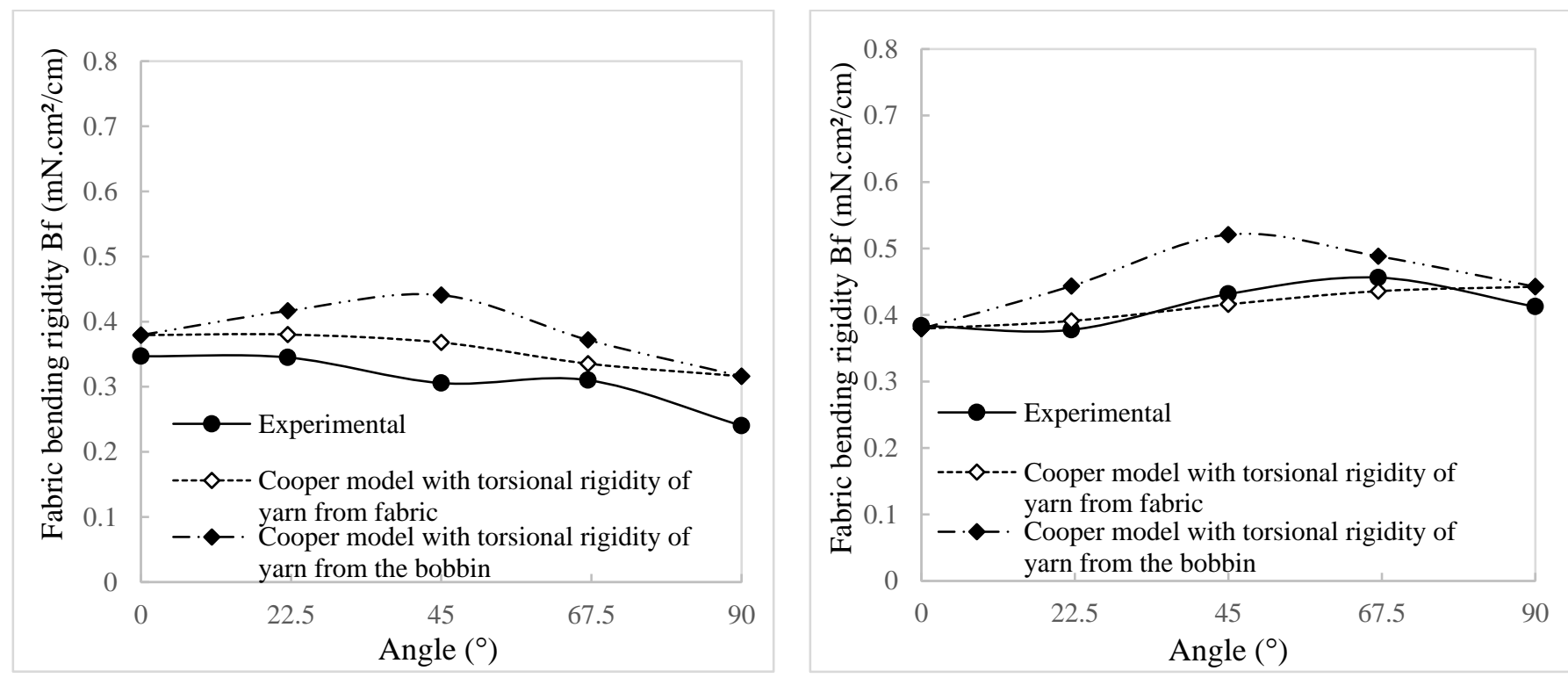
Figure 8. Comparison of experimental and calculated bending rigidities for Sample A

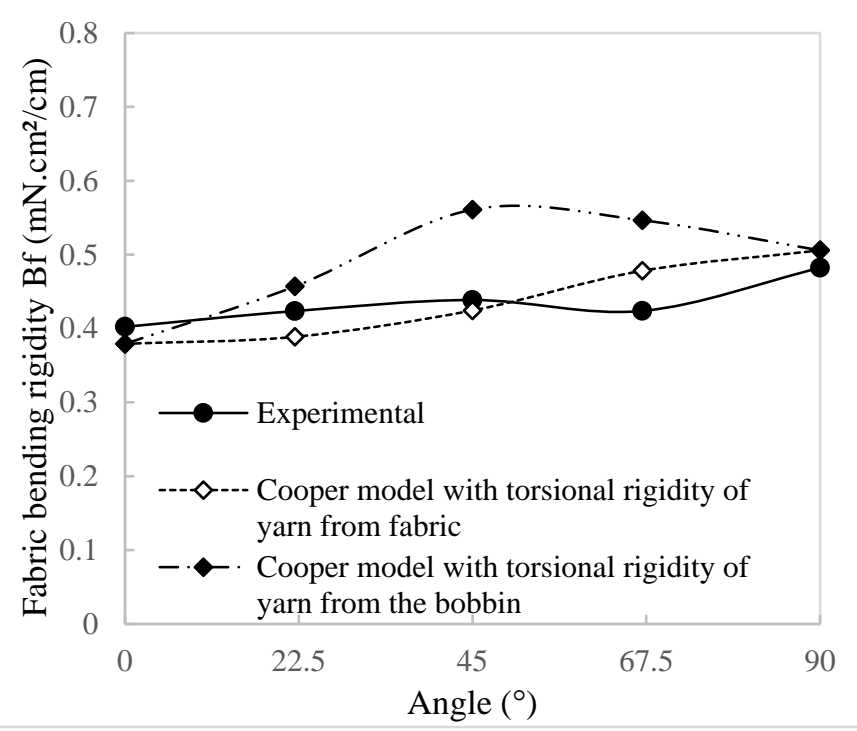

Figure 10. Comparison of experimental and calculated bending rigidities for Sample C

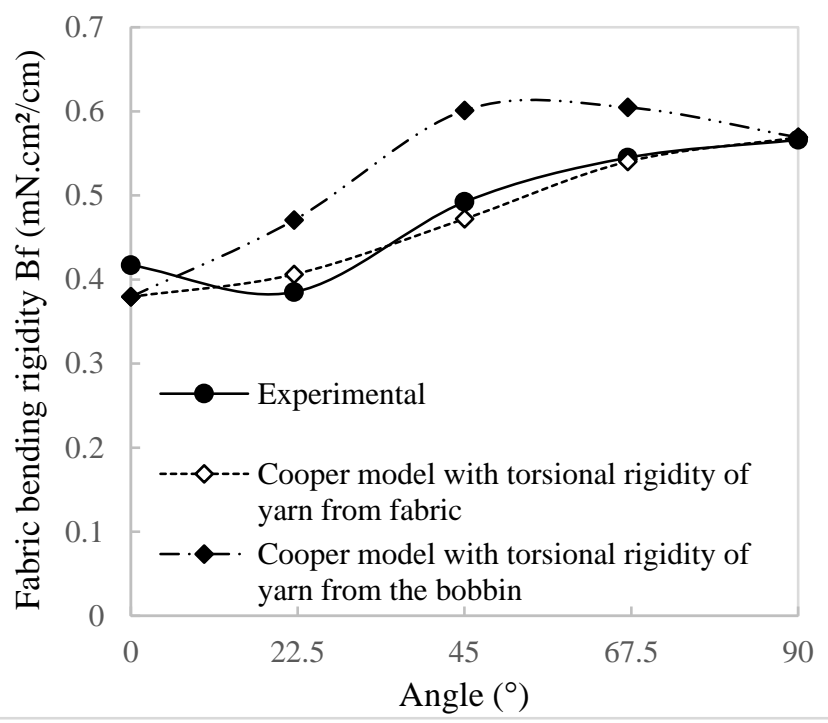

Figure 12. Comparison of experimental and calculated bending rigidities for Sample E

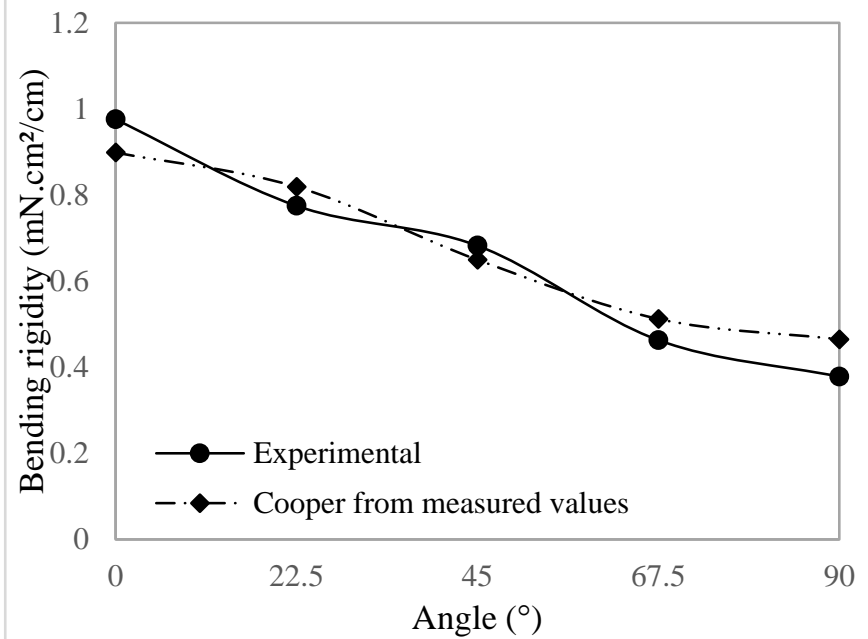

Figure 14. Comparison of experimental and calculated bending rigidities for Sample $G$
Figure 9. Comparison of experimental and calculated bending rigidities for Sample B

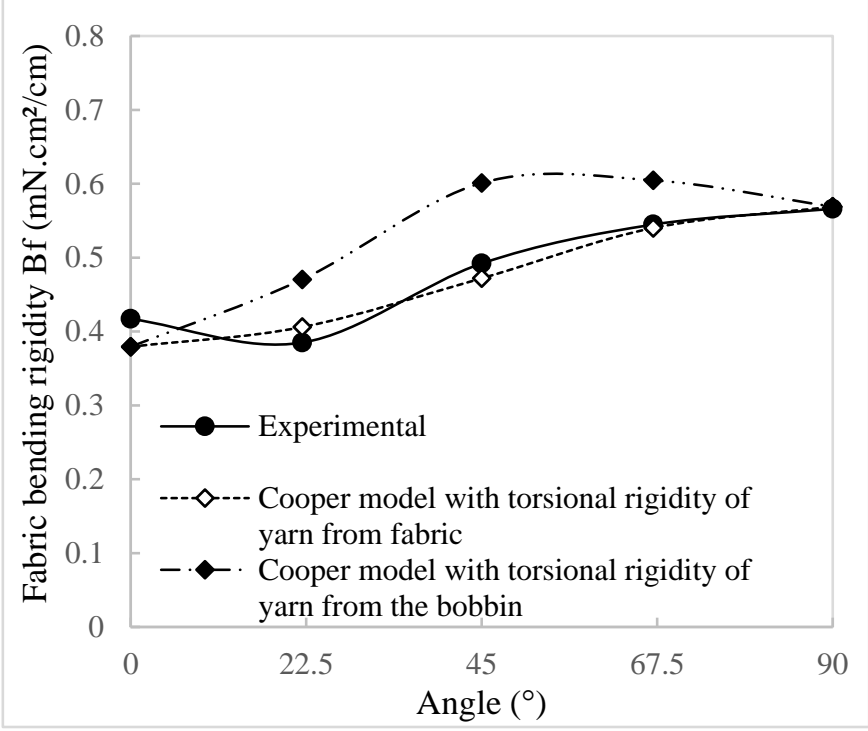

Figure 11. Comparison of experimental and calculated bending rigidities for Sample D

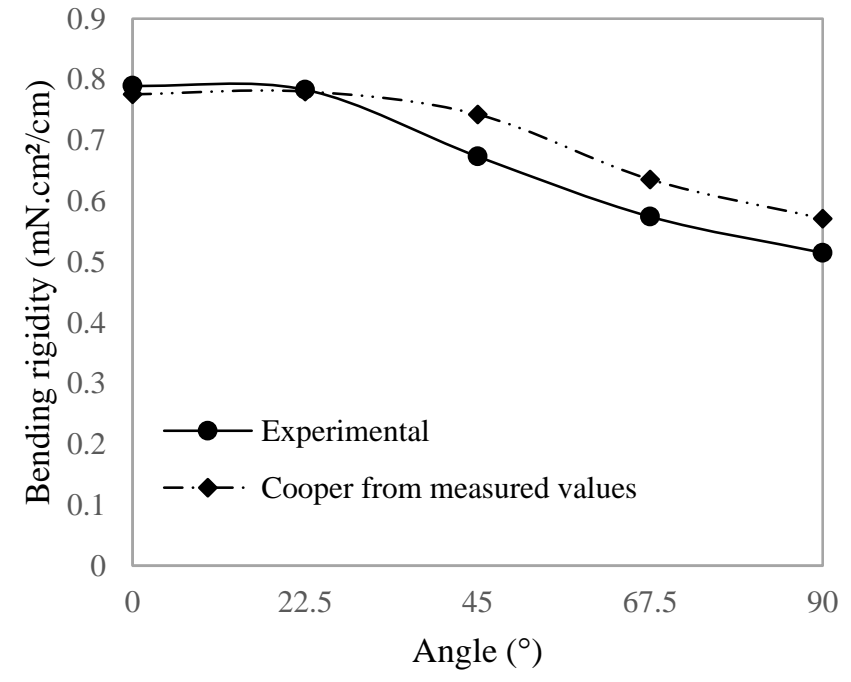

Figure 13. Comparison of experimental and calculated bending rigidities for Sample F

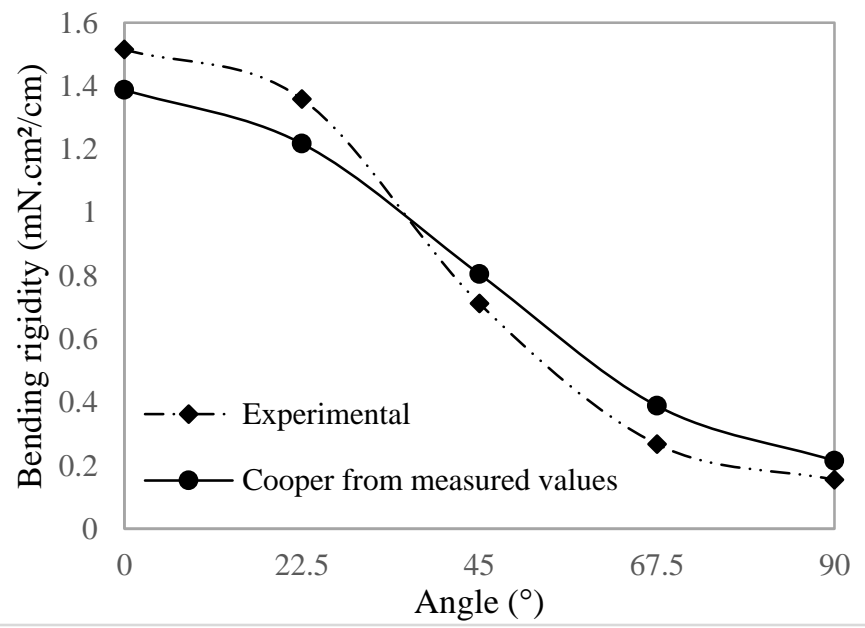

Figure 15. Comparison of experimental and calculated bending rigidities for Sample $\mathrm{H}$ 


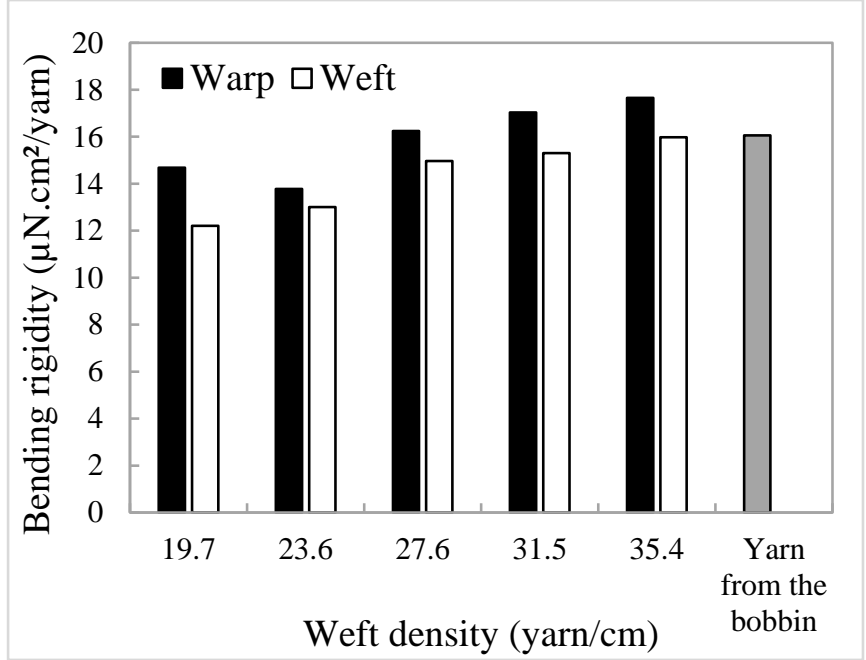

Figure 16. Bending rigidity per yarn calculated from fabric bending rigidity and measured yarn bending rigidity from the bobbin for Samples A to E

\section{Conclusion}

The effect of yarn torsional rigidity on the Cooper model for fabric bending rigidities in any direction was verified. Five cotton fabrics were made and tested along with three commercial samples in experiments. The torsional rigidities of yarn from the bobbin and yarn directly extracted from the fabric were measured with a yarn torsional tester. The bending rigidity of yarn from the bobbin was measured using the same pure bending tester used for fabric bending testing. The fabric bending rigidity calculated using torsional rigidities of yarns extracted from the fabric showed better agreement with the experimental values than that calculated using the torsional rigidity of yarn from the bobbin. Measurements showed that the torsional rigidity of yarn from the bobbin was appreciably higher than torsional rigidities of yarns from fabric. This could be due to the crimp of the yarn. The results show that the torsional rigidity of the yarn is affected by the weaving process and does not remain the same as that of yarn on the bobbin. It was thus found that, in predicting the bending rigidity of fabric using the Cooper model, themeasured torsional rigidities of yarns extracted from fabric should be used instead of yarn straight from the bobbin. The same results were obtained for the commercial fabrics. These results will be useful in fabric simulations.

\section{Acknowledgements}

This work was supported by JSPS KAKENHI under grant numbers JP24220012.

\section{References}

1. Pierce FT. The "handle" of cloth as a measurable quantity. J Textil Inst 1930; 21: T377-T416.

2. Grosberg P. The mechanical properties of woven fabrics-Part ii: The bending of woven fabrics. Text Res $J$ 1966; 36(3): T205-T211.

3. Go Y, Shinohara A and Matsuhashi F (1958). Viscoelastic studies of textile fabric (V) On the relationship between the bending behaviours of fabrics and fabric structures. Sen'i Gakkaishi 1958; 14(2), 78-83.

4. Cooper DNE. 24—The stiffness of woven textiles. J Textil Inst 1960; 51(8): T317-T335.

5. Chapman BM and Hearle JWS. The bending and creasing of multicomponent viscoelastic fibre assemblies. J Textil Ins 1971; 63(7): T385-T412.

6. Shinohara F, Shinohara A, and Sakaebara K. A theoretical study on the anisotropy of the bending rigidity of woven fabrics. J Textil Mach Soc Jpn 1979; 32(8): T66-T71.

7. Saxl IJ. The quantitative determination of stiffness in individual yarns. Text Res $J$ 1936; 6(3): 152-156. 
8. Horio, M. and Onogi, S. Forced vibration of reed as a method of determining viscoelasticity. J Appl Phys 1951; 22(7): 977-981.

9. Isshi T. Bending tester for fibers, yarns and fabrics. J Textil Mach Soc Jpn 1957; 3(2): 48-52.

10. Kawabata S. The standardization and analysis of hand evaluation. 2nd ed. Osaka: Textile Machinery Society of Japan, 1980.

11. Postle R, Burton P and Chaikin M. 41-The torque in twisted single yarn. J Textil Inst Trans 1964; 55(9): T448-T461.

12. Denney FW, Platt MM and Butterworth GAM. Mechanics of elastic performance of textile materials. Part XVII: Torsional recovery of filaments and singles yarns, and plied-yarn balance. Text Res J 1966; 36(1): $12-$ 30.

13. Kimura H, Matsumoto M, Matsumoto YI and Fukushima K. Structure of novel composite single yarn from polypropylene staple fibers. J Text Eng 2012; 58(3), 27-34.

14. Lo WS, Choi KF and Lo TY. Measurement of yarn bending and torsion rigidities of naturally curved yarns. Part 1: Monofilament yarn in helical shape. Text Res J 2010; 80(18): 1875-1886.

15. Hoi WY. A study of yarn torsional property and its effects on knitted fabric. PhD Thesis, Institute of Textile and Clothing, The Hong Kong Polytechnic University, Hong Kong, 2011.

16. Inui $\mathrm{S}$, Okabe $\mathrm{H}$, Takatera $\mathrm{M}$, Hashimoto $\mathrm{M}$ and Horiba $\mathrm{Y}$. A fabric simulation based on a model constructed from a lower level. In: Computational Engineering in Systems Applications, IMACS Multiconference on, Vol. 1, pp. 178-180, IEEE, 2006.

17. Xiaoqun D, Li YI and Zhang X. Simulating anisotropic woven fabric deformation with a new particle model. Text Res J 2003; 73(12): 1091-1099.

18. Xiao $\mathrm{M}$ and Geng Z. A model of rigid bodies for plain-weave fabrics based on the dynamics of multibody systems. Text Res J 2010.

19. Inui S, Mesuda Y, Horiba Y and Kurihara M. Fabric weave form simulation based on the characteristics of yarn. Biometrics and Kansei Engineering (ICBAKE), 2011 International Conference on. IEEE, 2011.

20. Zhou G, Sun X and Wang Y. Multi-chain digital element analysis in textile mechanics. Compos Sci Technol 2004; 64(2): 239-244.

21. JIS. Handbook 54 Sen'i. Japan: Japanese Standards Association, 2010. 